\title{
Efficient Search Method of Deperming Protocol for Magnetic Silence of Vessel
}

\author{
Jong-Wang Kim ${ }^{1}$, Sang-Hyun Kim ${ }^{1}$, Ji-ho Kim ${ }^{1}$, Hyang-Beom Lee ${ }^{1 *}$, and Hyun-Ju Chung ${ }^{2}$ \\ ${ }^{1}$ Soongsil University, Seoul 06978, Korea \\ ${ }^{2}$ Agency for Defense Development, Changwon 51691, Korea
}

(Received 30 August 2016, Received in final form 23 February 2017, Accepted 13 March 2017)

\begin{abstract}
This research is proposed with the efficient searching method of deperming protocol for magnetic silence. The deperming protocol has been changed depending on the initial magnetic field, the final magnetic field, and the shot number, so deciding parameter is highly important. Therefore, in this paper, the value of the initial magnetic field is supposed to be fixed to that of the saturation field, and excluding the initial magnetic field in the variable, the deperming protocol has been analyzed depending on the final magnetic field and the change of the shot number. In the result of this experiment, the final magnetic field gets high performance to the reverse of the direction of the initial magnetization, and it is resulted that the shot number inverse proportion to the size of the final magnetic field.
\end{abstract}

Keywords : deperming, anhysteretic, magnetic silence

\section{Introduction}

Recently, marine wars tend to be changing from the battle of radar to that of stealth. Stealth technique is one of the vessel detection technology which neutralizes radar, which is needed to both advanced countries where radar technique is saturated and developing countries for which neutralize radar technique in advanced countries. So, stealth technique should minimize infrared rays, a noise, and magnetic signal occurring in vessel. In particular, vessel cannot help being weak to magnetic signal, because it mainly consists of ferromagnetic material. Magnetic signal is the one of the convenient methods of searching vessel, because of not being metal materials in the ocean unlike land. Because of development IT technology and improving of sensor function, it is able to be measured into nT unit and magnetic mine and torpedo become a huge threat element to vessel sail. During the last 50 years, actually, there's an over $78 \%$ damage from magnetic mine of entire damage in the U.S. vessel. The price of magnetic mine is thousands of dollars, as well as the repair price from a damage is tens of thousands, many countries have adopted magnetic mine and used it because of highly efficiency on the cost. For the stable vessel sail

(C)The Korean Magnetics Society. All rights reserved.

*Corresponding author: Tel: +82-2-820-0641

Fax: +82-2-817-7961, e-mail: hyang@ssu.ac.kr from this threat, magnetic signal occurring vessel should be minimized and realized magnetic silence $[1,2]$.

\section{Magnetic Silence}

Magnetic silence is the method doesn't disrupt magnetic field with magnetic signal minimizing. Magnetic silence is divided into degaussing method that reduces induced magnetization element by the earth magnetic field and deperming method that reduces residual magnetization element in vessel. Degaussing method is that minimizes induced magnetization element occurring vessel by winding a coil to 3 -axis direction in vessel and generating magnetic field to the reverse of induced magnetization element. It measures the value of electric current on coil in 3-axis direction by prediction induced magnetization of vessel on the earth magnetic field on the basis of the measured data in magnetic treatment facility [3].

Deperming method is that minimizes the residual magnetization element in vessel according to cycling to an amount of reducing gradually B-H curve. It, unlike degaussing method, should approve the magnetic field to nonlinear field of B-H curve, and there's a difficulty to apply deperming protocol according to nonlinear property [4].

\subsection{Cause of Occurring the Residual Magnetization Element}

Residual magnetization element occurs by the move of 


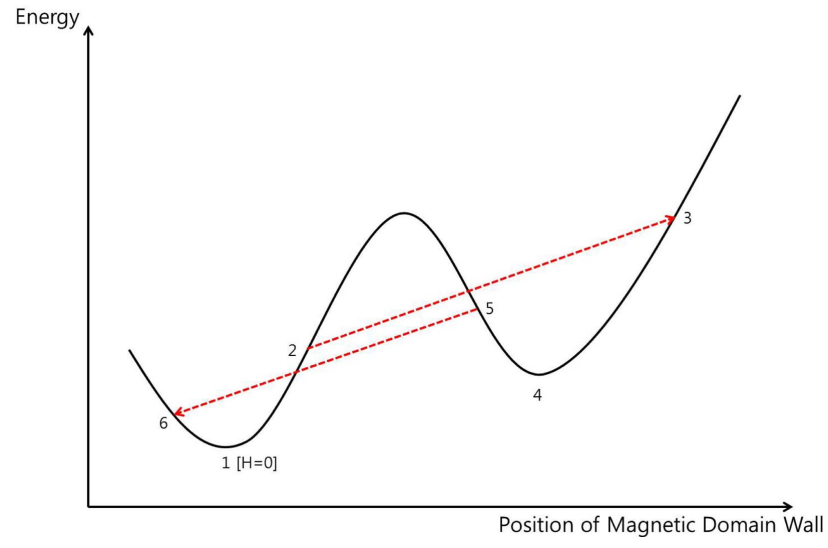

Fig. 1. (Color online) Reversible and irreversible move of magnetic domain wall.

reversible and irreversible of magnetic domain wall existing ferromagnetic. Figure 1 is the graph about energy on the position of magnetic domain wall. When position 1 is designed to be minimum energy and is approved to $\mathrm{H}$, magnetic domain wall would move reversibly to position 2 and come back to position 1 as magnetic field is on 0 . If magnetic field would be sufficient as it moves magnetic domain wall to position 2 at the maximum energy slope, magnetic domain wall jumps irreversibly to position 2, which is called Barkhausen Jump. At this point, magnetic field is designed to be reduced on 0 , magnetic domain wall would go back to not position 1 but position 4, residual magnetization elements would occur with energy at position 4. In order to come back to position 1, the magnetic field in reverse direction should be approved as Barkhausen Jump from position 5 to 6 [9].

\subsection{Deperming Protocol}

Deperming protocol is the method that applies H Field in vessel such as Fig. 2. The initial magnetic field is designed with magnetization of inner vessel to be fully arranged in a longitudinal direction, and magnetization in vessel is designed in a same situation whenever deperming protocol is applied. Therefore, the initial magnetic field is supposed to have a sufficient amount as vessel is saturated. The amount of reduction magnetic field is the parameter that decides the size of dividing magnetic domain. The smaller the amount of reduction magnetic field is, the more detailed the field of magnetic domain divides. Shot applied time should be fully applied considering magnetic viscosity characteristic of ferromagnetic, and dead time is considered about the temperature increase of deperming coil [7].

As applying deperming protocol, irreversible move of magnetic domain wall would occur. From Fig. 3 to 9 refers

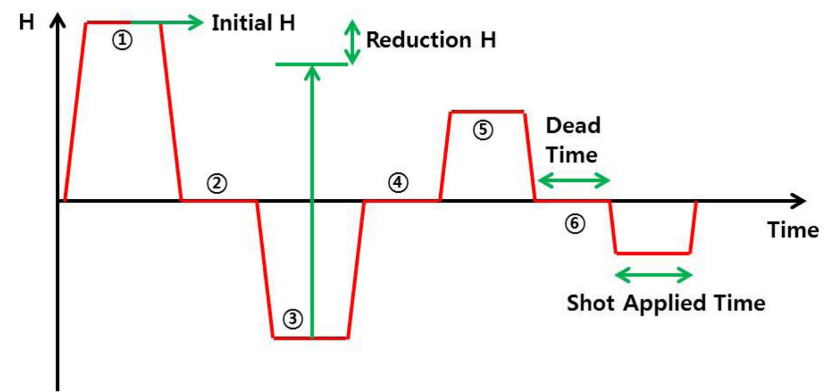

Fig. 2. (Color online) Deperming protocol.

to position of magnetic domain wall, magnetization direction, and the amount of energy as applying deperming protocol. Figure 3 shows the initial state before applying deperming protocol. Such as (1) in Fig. 2, External $\mathrm{H}$ Field is designed to be applied, and Barkhausen Jump would occur in Fig. 4. Though External H Field is designed to be applied with the same amount, the size of Internal $\mathrm{H}$ Field should be different because of demagnetizing field on the shape of vessel, as well as different size in energy is supposed to have depending on the position of

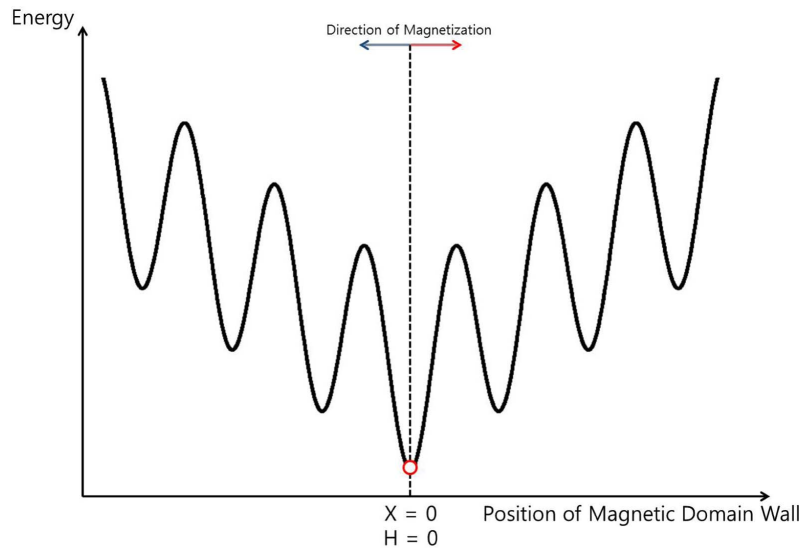

Fig. 3. (Color online) Initial magnetization states.

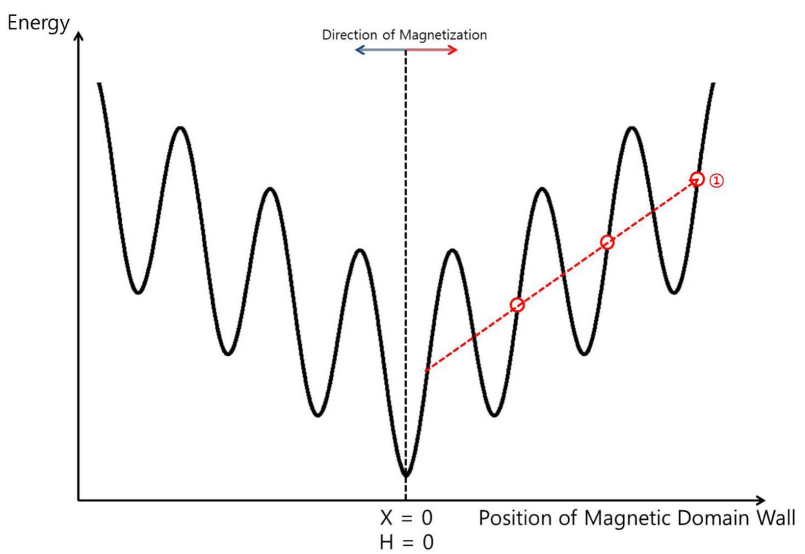

Fig. 4. (Color online) Magnetization states at point (1). 


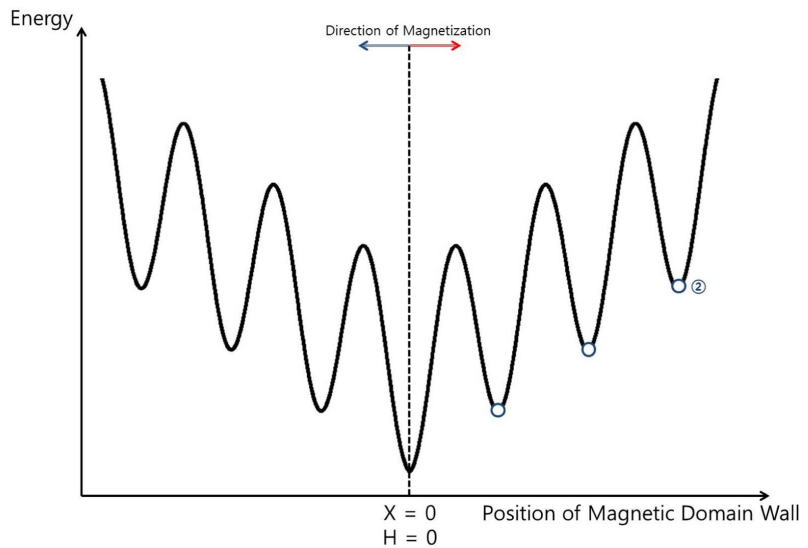

Fig. 5. (Color online) Magnetization states at point (2).

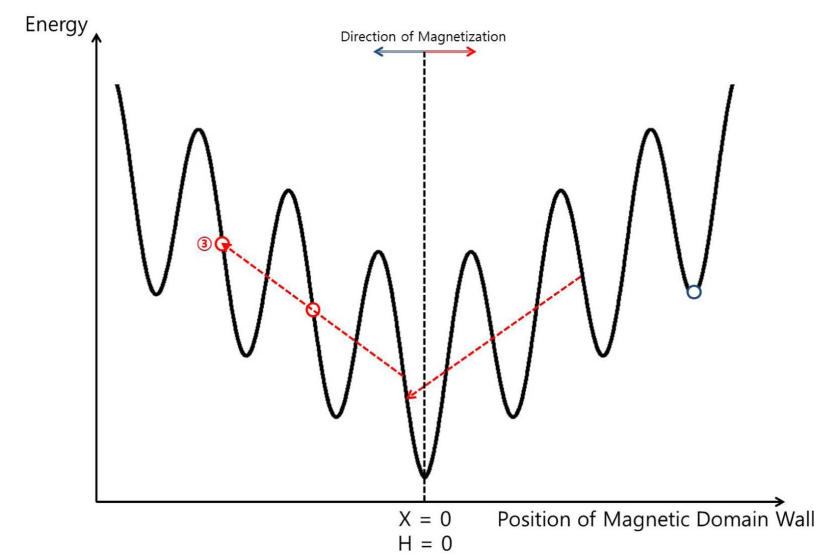

Fig. 6. (Color online) Magnetization states at point (3) .

magnetic wall because inner impurities disrupt move of magnetic domain wall. When External H Field is removed such as (2) in Fig. 2, it comes in stable energy state. At this time, as the direction of all residual magnetization element is like $\rightarrow$, the magnetization direction is also like $\rightarrow$. If External $\mathrm{H}$ Field is designed to be applied in the

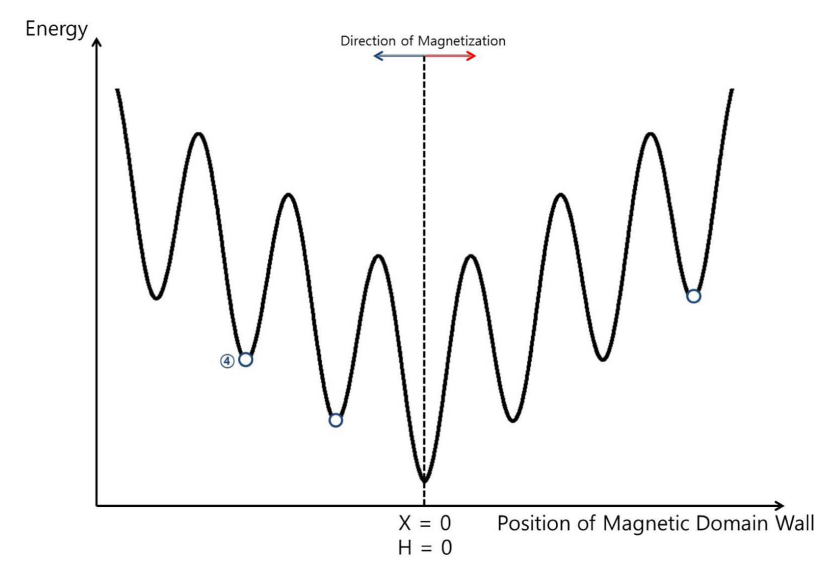

Fig. 7. (Color online) Magnetization states at point (4).

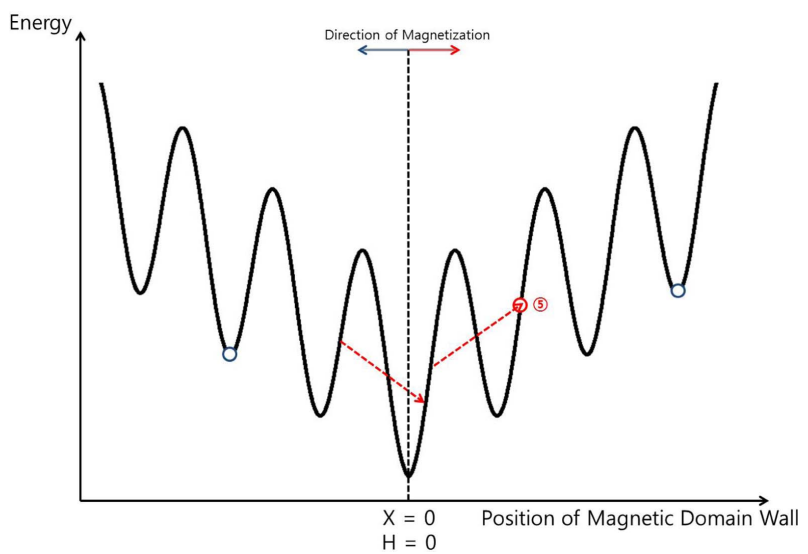

Fig. 8. (Color online) Magnetization states at point (5).

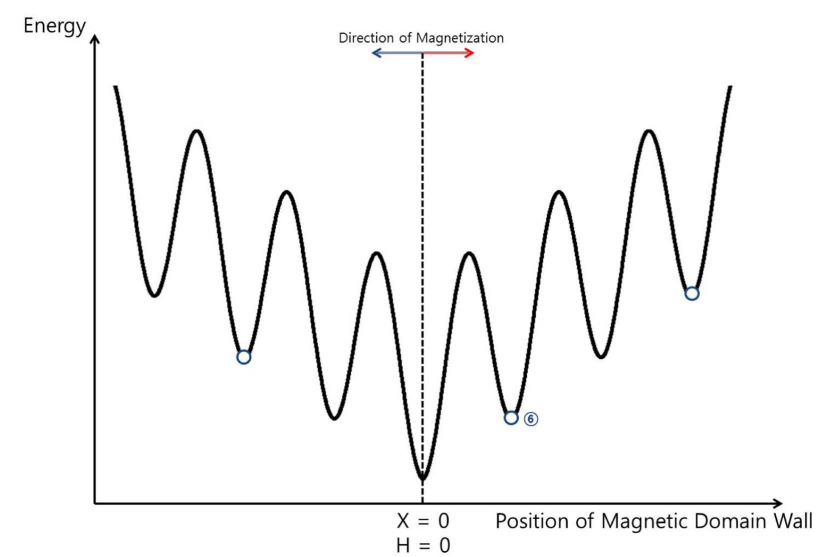

Fig. 9. (Color online) Magnetization states at point (6) .

reverse direction such as (3) which is less than (1) such as Fig. 2, all magnetization element would not be reverse to the direction like $\rightarrow$. Therefore, some magnetization would be reverse such as Fig. 6, if External H Field is designed to be removed, it would come in stable energy state such as Fig. 7. Figure 9 shows residual magnetization of vessel after the end of deperming protocol, and the magnetization direction would be decided by the sum of residual magnetization element in direction like $\leftarrow$ and direction like $\rightarrow$.

\section{Deperming System}

\subsection{Small Specimen}

This paper is designed with small specimen of submarine model such as Fig. 10. for deperming experiment. The length of specimen is $330 \mathrm{~mm}$, external diameter 44 $\mathrm{mm}$, internal diameter $35 \mathrm{~mm}$, and SM45C carbon steelmaterials. Figure 11 refers to B-H Curve of SM45C. The saturation magnetic field strength of SM45C-materlas is $4490 \mathrm{~A} / \mathrm{m}$. 


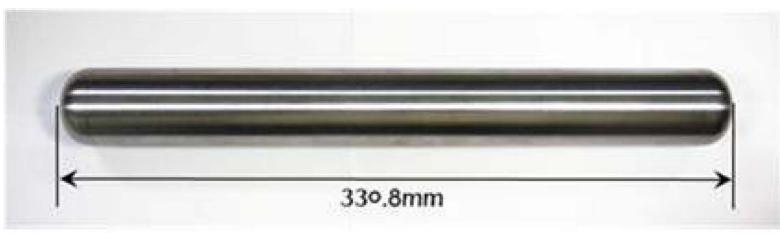

Fig. 10. (Color online) Small specimen.

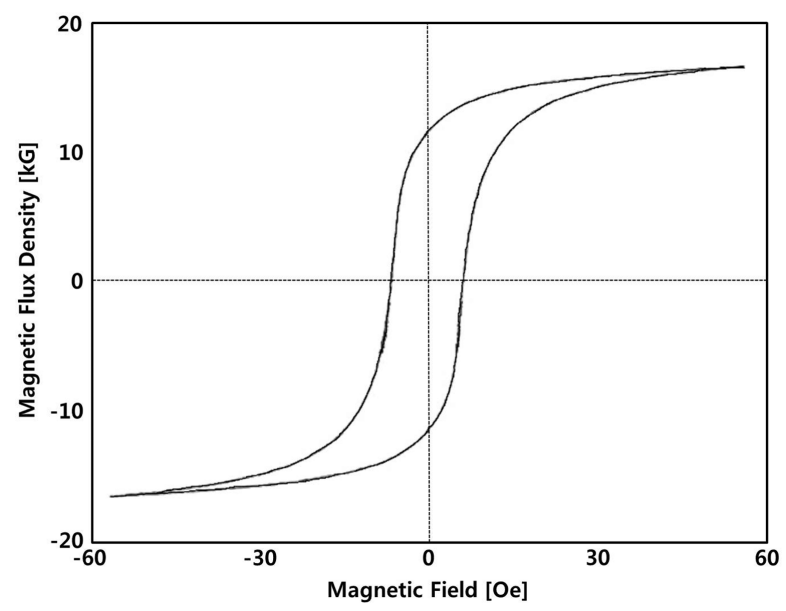

Fig. 11. SM45C B-H curve.

\subsection{Small Deperming Coil}

In order to apply deperming protocol into small specimen $\mathrm{Z}$ coil and $\mathrm{X}$ coil are supposed to be needed, and $\mathrm{Z}$ coil is able to compensate magnetic field in vertical direction and $\mathrm{X}$ coil to compensate magnetic field in horizontal direction and apply deperming protocol. As $\mathrm{Z}$ coil is needed with weak magnetic field in wide area, it is designed with square Helmholtz coil. As X coil is needed with strong magnetic field in small area, it is designed with cylinder solenoid coil. Figure 12 shows the manufactured small deperming coil.

\subsection{Magnetic Field Measurement System}

This paper is designed with measurement system such as Fig. 13 in order to measure magnetic field of small

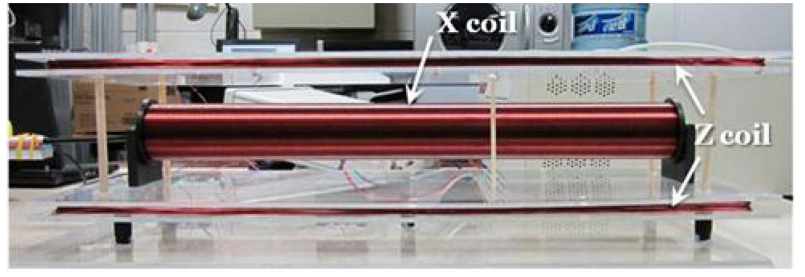

Fig. 12. (Color online) The manufactured deperming coil.

Table 1. Mag-03 flux gate magnetic sensor.

\begin{tabular}{ccc}
\hline \hline Factor & Value & Unit \\
\hline Measuring Range & \pm 70 & $\mu \mathrm{T}$ \\
Scaling & 143 & $\mathrm{mV} / \mu \mathrm{T}$ \\
Resolution & 0.534 & $\mathrm{nT}$ \\
Offset Error & \pm 5 & $\mathrm{nT}$ \\
Scaling Temperature Coefficient & +5 & $\mathrm{ppm} /{ }^{\circ} \mathrm{C}$ \\
Offset Temperature Coefficient & \pm 0.1 & $\mathrm{nT} /{ }^{\circ} \mathrm{C}$ \\
\hline
\end{tabular}

specimen. Magnetic field measurement system is supposed to move small specimen by using Rail, Moving Cart, and Motor, and magnetic field of small specimen is supposed to be measured by Flux Gate Sensor. The order of measuring magnetic field occurring on specimen is as follows.

(1) Applying magnetization/deperming protocol on specimen

(2) Moving specimen to the end of left and right of rail

(3) Applying offset to zero function of magnetic measurement sensor

(4) Maneuver a round-trip specimen to the left and right

(5) Measuring the distance of specimen and size of magnetic field

Table 1 shows specifications of Flux Gate type ThreeAxis Magnetic Sensor Mag-03 on Bartington Instruments

\section{Result of Experiment}

\subsection{Initial Magnetization}

The initial magnetization element is supposed to be

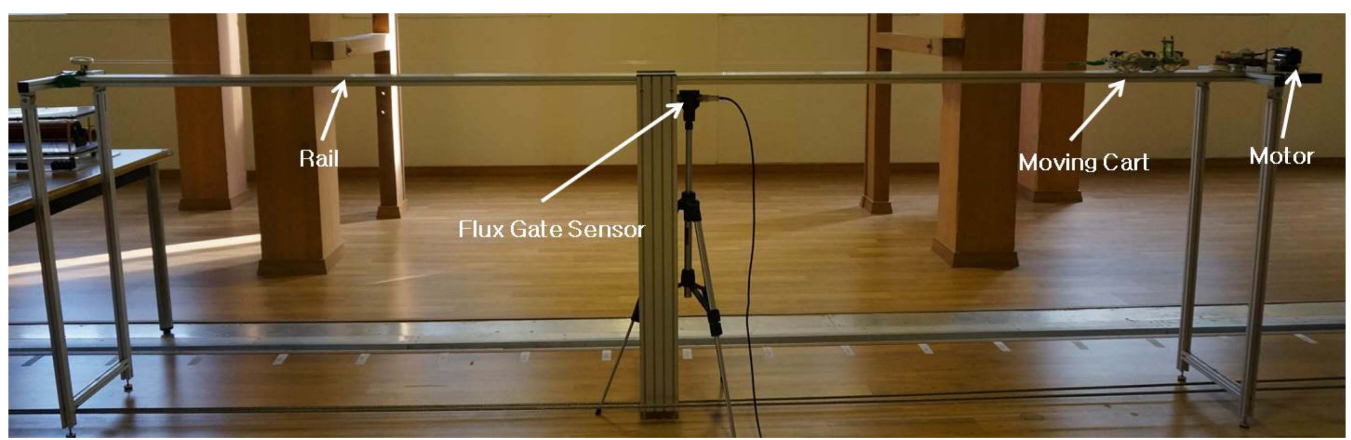

Fig. 13. (Color online) Measurement system of magnetic field. 


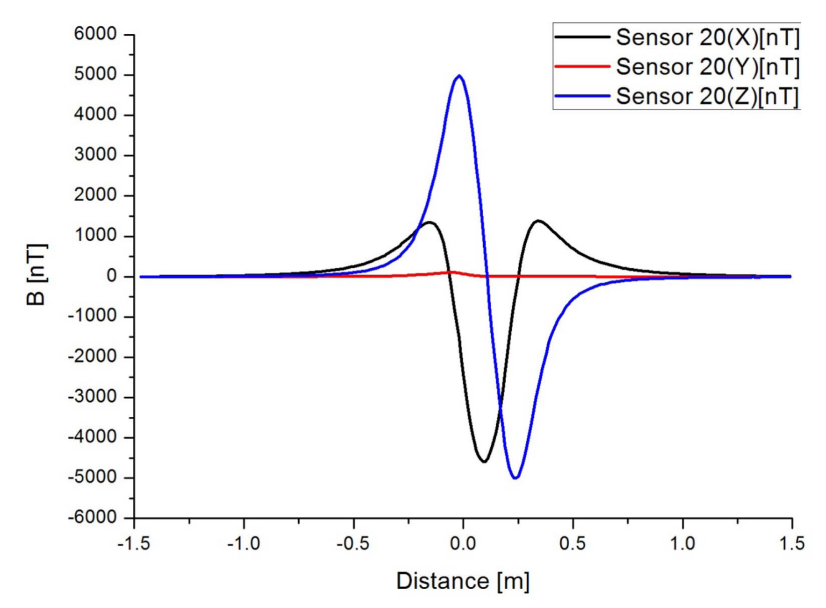

Fig. 14. (Color online) Initial magnetization.

always same in order to apply deperming protocol. As consequences, in this paper, specimen applying magnetic field of $+520 \mathrm{~A} / \mathrm{m}$ for $100 \mathrm{sec}$. under $\mathrm{X}$ coil and $\mathrm{Z}$ coil being the same of the size of earth's magnetic field and offset $\mathrm{H}$ field of different distance is magnetized such as Fig. 14. At this time, the distance between the center of specimen and magnetic field measurement sensor is 16 $\mathrm{cm}$, and $\mathrm{x}$-axis shows the coordinate on specimen moving.

\subsection{Anhysteretic Deperming Protocol}

Anhystereitic Deperming Protocol such as Fig. 15 is the method to reduce $\mathrm{H}$ Field at equidistant intervals. Deperming function, in this paper, is measured by changing final magnetic field and shot number in the state of fixing on $1040 \mathrm{~A} / \mathrm{m}$ at initial magnetic field. Table 2 shows Anhysteretic Deperming Protocol depending on final magnetic field and shot number.

\subsection{Result of Experiment}

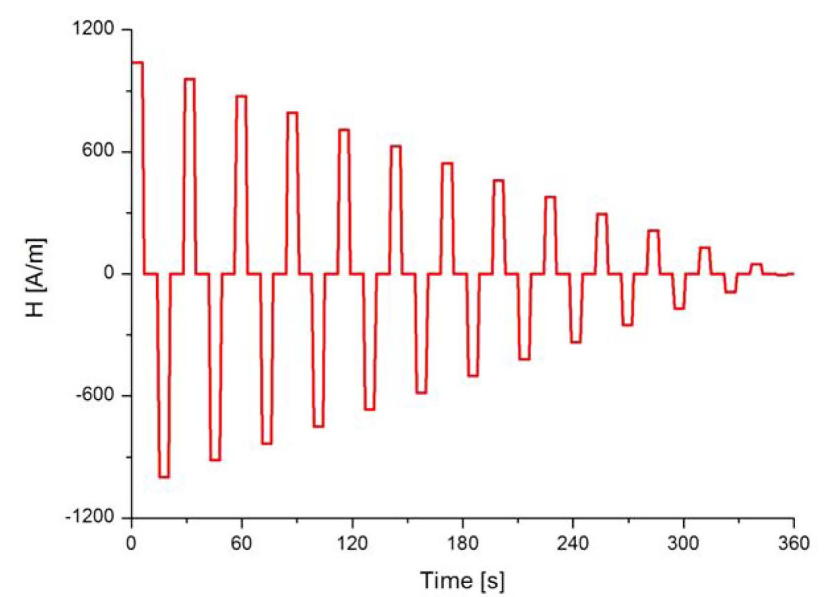

Fig. 15. (Color online) Anhysteretic deperming protocol.
Table 2. Anhysteretic deperming protocol on shot number and final magnetic field.

\begin{tabular}{cccc}
\hline \hline \multirow{2}{*}{ Shot No. } & $\begin{array}{c}\text { Initial H } \\
{[\mathrm{A} / \mathrm{m}]}\end{array}$ & $\begin{array}{c}\text { Reduction } \mathrm{H} \\
{[\mathrm{A} / \mathrm{m}]}\end{array}$ & $\begin{array}{c}\text { Final H } \\
{[\mathrm{A} / \mathrm{m}]}\end{array}$ \\
\hline \multirow{4}{*}{12} & 92.8902 & -18.2 \\
& & 85.0902 & -104.0 \\
& \multirow{3}{*}{1040} & 80.3634 & -156.0 \\
& & 78.0000 & -182.0 \\
& & 73.2732 & -234.0 \\
\hline \multirow{3}{*}{24} & & 44.4262 & -18.2 \\
& & 44.0882 & -26.0 \\
& & 42.9572 & -52.0 \\
& & 40.6952 & -104.0 \\
& & 39.0000 & -143.0 \\
& & 38.4358 & -156.0 \\
& & 35.0428 & -234.0 \\
\hline \multirow{3}{*}{36} & & 29.1954 & -18.2 \\
& & 28.9718 & -26.0 \\
& & 28.2282 & -52.0 \\
& & 27.4846 & -78.0 \\
& & 26.7436 & -104.0 \\
& & 26.0000 & -130.0 \\
& & 25.6282 & -143.0 \\
& & 25.2564 & -156.0 \\
& & 24.5154 & -182.0 \\
& & & -208.0 \\
\hline \multirow{6}{*}{1040} & &
\end{tabular}

Figure 16 shows $\mathrm{Bx}$, and $\mathrm{Bz}$ element on changing the size of final magnetic field of Anhysteretic Deperming Protocol at 36 shot. Figure 17 to Fig. 20 is the measured magnetic field signal of small specimen in each -280 , $-156,-130$, and $-104 \mathrm{~A} / \mathrm{m}$ of final magnetic field. At $-280 \mathrm{~A} / \mathrm{m}$ of final magnetic field, magnetization significantly results in reverse direction for initial magnetization. On the basis of this result, if the size of final

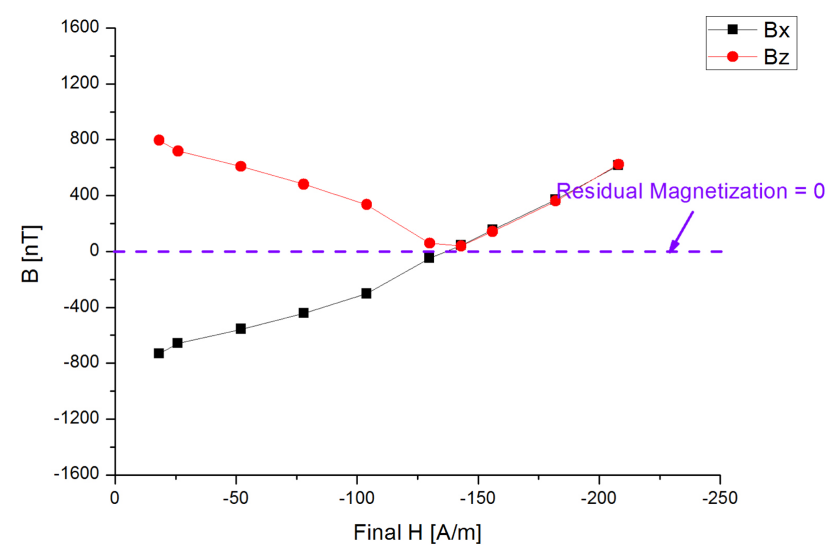

Fig. 16. (Color online) Magnetic field on changing final magnetic field (36 Shot). 


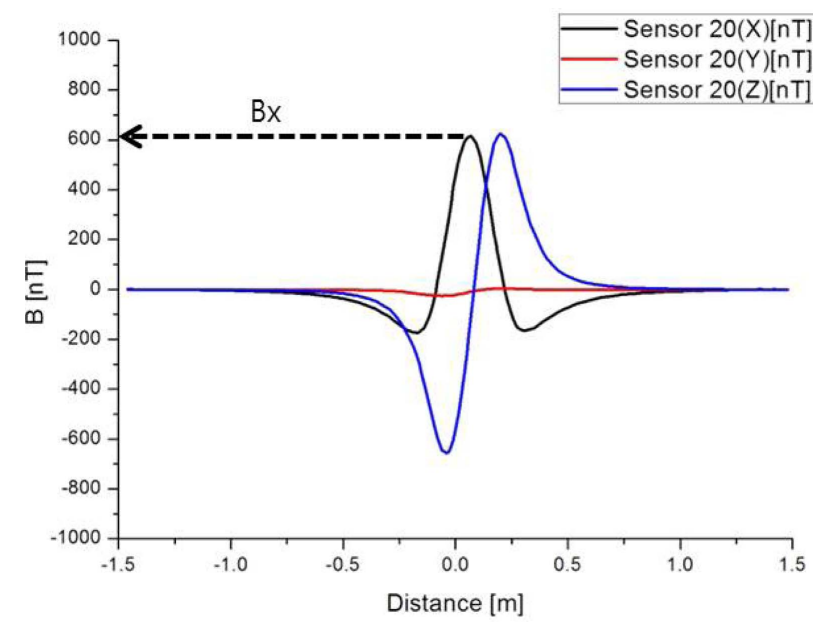

Fig. 17. (Color online) Magnetic field measurement data (Final $\mathrm{H}=-208 \mathrm{~A} / \mathrm{m})$.

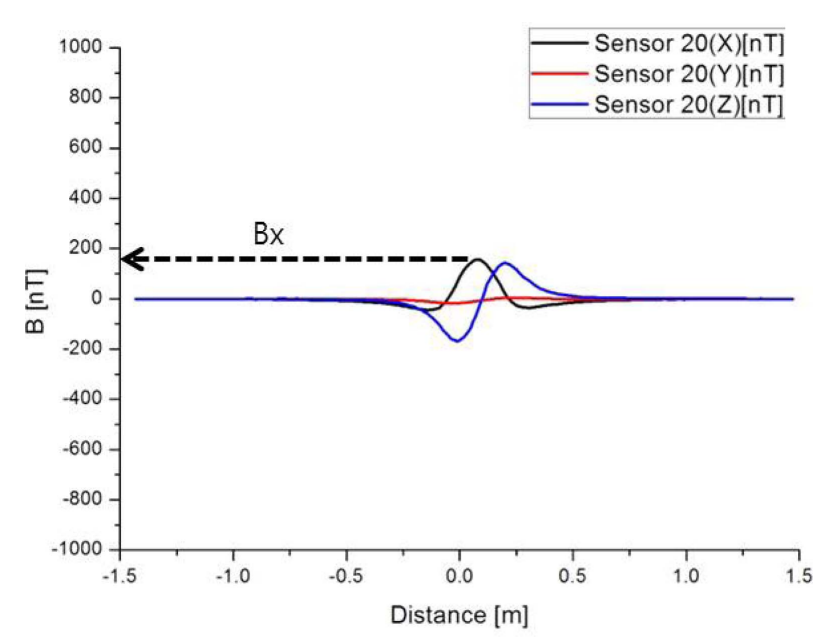

Fig. 18. (Color online) Magnetic field measurement data (Final $\mathrm{H}=-156 \mathrm{~A} / \mathrm{m}$ ).

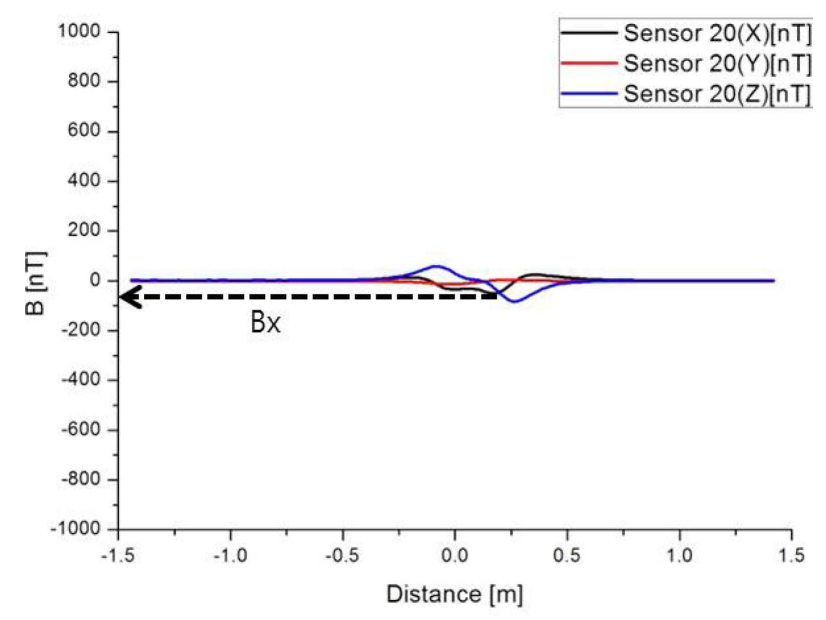

Fig. 19. (Color online) Magnetic field measurement data (Final $\mathrm{H}=-130 \mathrm{~A} / \mathrm{m})$.

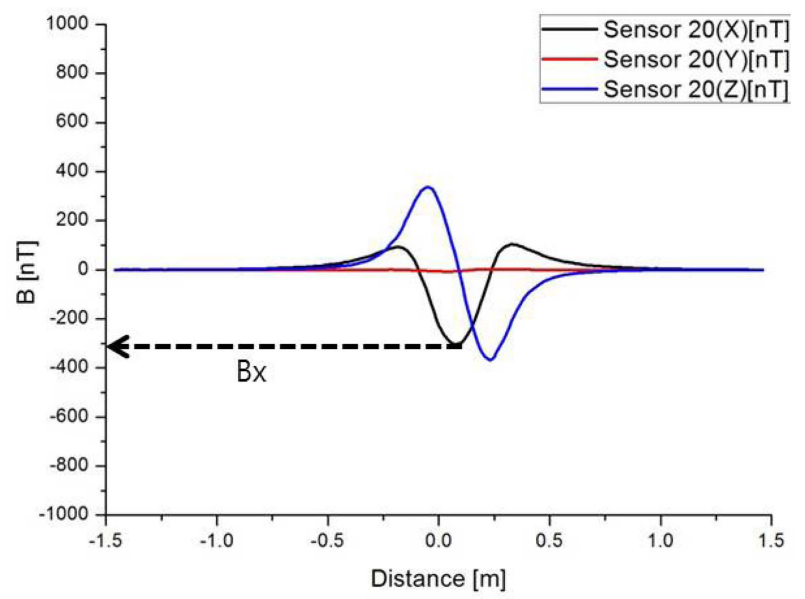

Fig. 20. (Color online) Magnetic field measurement data (Final $\mathrm{H}=-104 \mathrm{~A} / \mathrm{m}$ ).

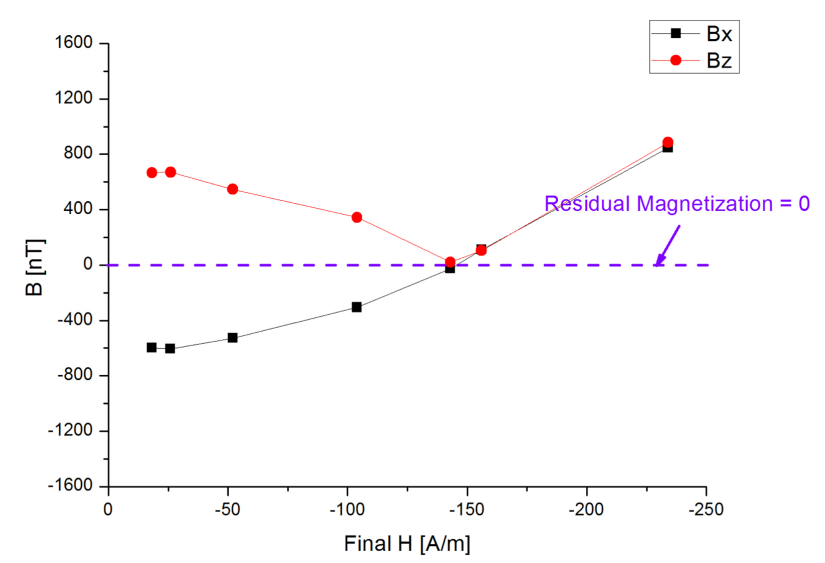

Fig. 21. (Color online) Magnetic field on changing final magnetic field (24 Shot).

magnetic field is reduced, it can be predicted that the sum of residual magnetization would be minimum after deperming protocol. By reducing the size of final magnetic field, as a consequence of measuring magnetic field of small specimen, the diction of magnetization is reversed at $-130 \mathrm{~A} / \mathrm{m}$ of final magnetic field, and it is resulted in that residual magnetization element is minimum.

Figure 21 and Fig. 22 is the graph of each $\mathrm{Bx}$ and $\mathrm{Bz}$ element on last magnetic size change of Anhysteretic Deperming Protocol at 24 shot and 12 shot. Magnetization direction, like at 36 shot Anhysteretic Deperming protocol, is supposed to be reverse on the basis of a certain final magnetic field, and residual magnetization element results in minimum.

Table 3 shows the measured data of magnetic field signal of small specimen on changing last current of Anhysteretic Deperming Protocol and shot number. In case at 12 shot Anhysteretic Deperming Protocol, mag- 


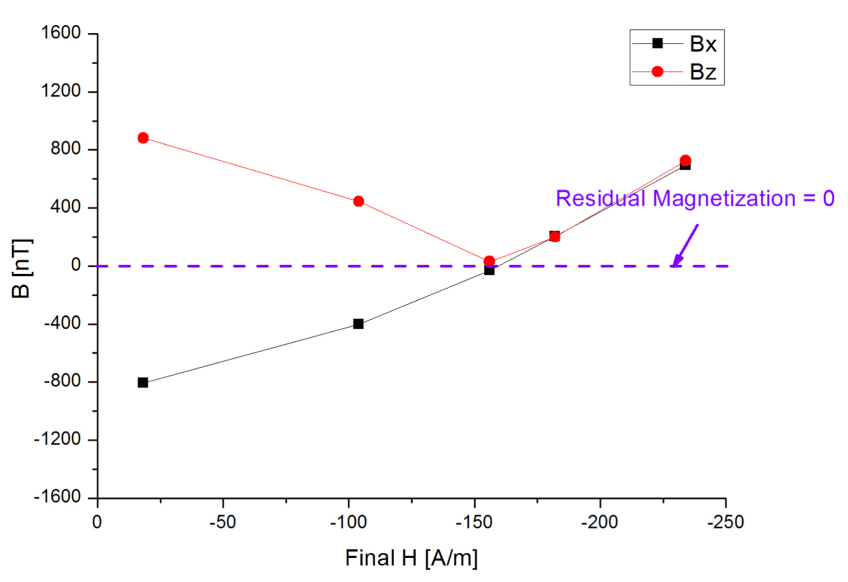

Fig. 22. (Color online) Magnetic field on changing final magnetic field (12 Shot).

netization direction is supposed to be reverse between $-150 \mathrm{~A} / \mathrm{m}$ to $-182 \mathrm{~A} / \mathrm{m}$, and in case at 24 shot, magnetization direction is supposed to be reverse $-130 \mathrm{~A} / \mathrm{m}$ to $-143 \mathrm{~A} / \mathrm{m}$.

\subsection{Efficient Search Method of Deperming Protocol} This paper is designed to experiment deperming performance by changing final magnetic field and shot

Table 3. Mangtic field $\mathrm{Bx}, \mathrm{Bz}$ element after deperming on final magnetic field and shot numner.

\begin{tabular}{cccc}
\hline \hline Shot No. & Fianl H $[\mathrm{A} / \mathrm{m}]$ & Bx $[\mathrm{nT}]$ & Bz $[\mathrm{nT}]$ \\
\hline \multirow{4}{*}{12} & -18.2 & -805.974 & 882.2383 \\
& -104 & -400.03 & 444.8624 \\
& -156 & -31.1068 & 30.65552 \\
& -182 & 203.4668 & 200.1342 \\
& -234 & 694.1618 & 725.5705 \\
\hline \multirow{4}{*}{24} & -18.2 & -600.229 & 667.2984 \\
& -26 & -605.798 & 670.7926 \\
& -52 & -526.892 & 546.0544 \\
& -104 & -306.994 & 343.8225 \\
& -143 & -26.6036 & 20.398 \\
& -156 & 111.6211 & 105.2992 \\
& -234 & 847.22 & 884.0778 \\
\hline \multirow{4}{*}{36} & -18.2 & -732.028 & 795.6914 \\
& -26 & -658.592 & 718.7154 \\
& -52 & -556.177 & 609.3326 \\
& -78 & -441.325 & 480.4859 \\
& -104 & -303.006 & 336.0305 \\
& -130 & -49.245 & 59.03147 \\
& -143 & 44.18078 & 40.01364 \\
& -156 & 156.7486 & 144.1365 \\
& -182 & 369.4811 & 361.1733 \\
& -208 & 615.8233 & 624.6234 \\
\hline \multirow{6}{*}{} & & &
\end{tabular}

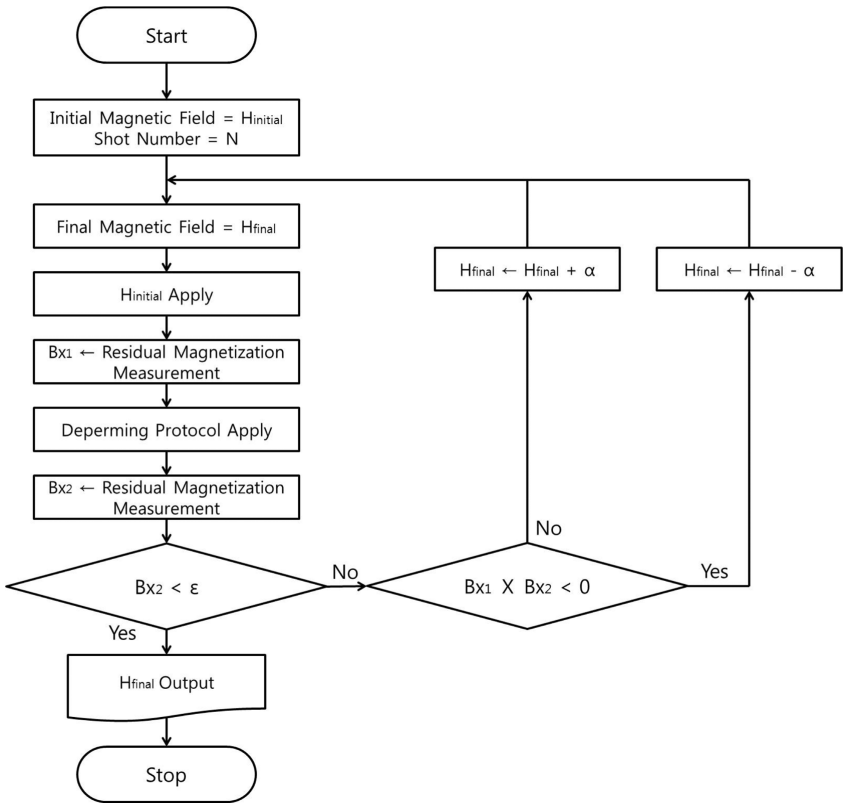

Fig. 23. Chart of efficient deperming protocol search.

number at a regular value of initial magnetic field. There, in this experiment result, exists the period that magnetic direction reverses on changing the size of final magnetic field, and it is resulted that residual magnetic element is in minimum between a reverse period of magnetization direction.

On the basis of experiment result in this paper, in case of magnetic direction reversing after applying initial magnetic direction and deperming protocol, it is supposed to reduce the size of final magnetic field, and the size of final magnetic field should be increased in case of same magnetization direction after applying deperming protocol. Figure 23 is the chart to shows efficient deperming protocol searching method.

\section{Conclusion}

This paper is designed to study about the efficient deperming protocol searching method for magnetic silence of vessel. For the experiment, is measured small specimen, small deperming coil and magnetic field measurement system, and by changing final magnetic field of Anhysteretic Deperming Protocol and shot number it is supposed to analyze deperming performance. At the fixed initial magnetic field and shot number, the highest deperming performance results at the point of reverse magnetization direction by changing final magnetic field, and it is resulted that shot number is in inverse with the size of final magnetic field. 


\section{Acknowledgement}

This work was supported by the Agency for Defense Development (Contract Number No. UD150009DD).

\section{References}

[1] John J. Holmes, Morgan \& Claypool.

[2] S. J. Davidson, T. Bailey, A. Parker, R. Twelvetrees, and M. Turener, Ultra Electronics PMES, United Kingdom (2000). [3] S. J. Davidson and G. J. Webb, Ultra Electronics PMES,
United Kingdom, 1998 (report).

[4] T. M. Baynes, G. J. Russell, and A. Bailey, Science \& Technology (2002).

[5] T. M. Baynes, G. J. Russell, and A. Bailey, IEEE Trans. Magn. 38, 1753 (2002).

[6] John J. Holmes, Naval Surface Warfare Center, USA (2006).

[7] R. Street and J. C. Woolley, IOPScience, 1949.

[8] Timothy Malcolm Baynes BSc The University of New South Wales, School of Physics, Faculty of Science.

[9] B. D. Cullity, Wiley (2001) pp 437-439. 